Expressions of the radius and the surface tension of surface of tension in terms of the pressure distribution for nanoscale liquid threads

This content has been downloaded from IOPscience. Please scroll down to see the full text. 2013 Chinese Phys. B 22126801

(http://iopscience.iop.org/1674-1056/22/12/126801)

View the table of contents for this issue, or go to the journal homepage for more

Download details:

IP Address: 159.226.199.8

This content was downloaded on 22/01/2014 at 09:48

Please note that terms and conditions apply. 


\title{
Expressions of the radius and the surface tension of surface of tension in terms of the pressure distribution for nanoscale liquid threads*
}

\author{
Yan Hong(阍 红) $)^{\mathrm{a} b)}$ ，Wei Jiu-An(魏久安) $)^{\mathrm{c})}$ ，Cui Shu-Wen(崔树稳) $)^{\mathrm{d})}$, and Zhu Ru-Zeng(朱如曾) $)^{\mathrm{b}) \dagger}$ \\ a) Department of Electronic Information and Physics, Changzhi University, Changzhi 046011, China \\ b) Key Laboratory of Microgravity, Institute of Mechanics, Chinese Academy of Sciences, Beijing 100190, China \\ c) Advanced Semiconductor Materials (ASM) Technology Singapore, 2 Yishun Avenue 7, Singapore, 768924 \\ d) Department of Physics and Electronic Information, Cangzhou Normal University, Cangzhou 061000, China
}

(Received 18 January 2013; revised manuscript received 17 May 2013)

\begin{abstract}
The expressions of the radius and the surface tension of surface of tension $R_{\mathrm{S}}$ and $\gamma_{\mathrm{s}}$ in terms of the pressure distribution for nanoscale liquid threads are of great importance for molecular dynamics (MD) simulations of the interfacial phenomena of nanoscale fluids; these two basic expressions are derived in this paper. Although these expressions were derived first in the literature [Kim B G, Lee J S, Han M H, and Park S, 2006 Nanoscale and Microscale Thermophysical Engineering, 10, 283] and used widely thereafter, the derivation is wrong both in logical structure and physical thought. In view of the importance of these basic expressions, the logic and physical mistakes appearing in that derivation are pointed out.
\end{abstract}

Keywords: nanoscale liquid thread, surface tension, surface of tension, pressure distribution

PACS: 68.03.Cd, 68.35.Md

\section{Introduction}

Molecular dynamics (MD) simulation is one of the useful methods to investigate the interfacial phenomena of nanoscale fluids, because it can provide detailed information on the molecular scale. ${ }^{[1,2]}$ To use MD simulation to compute the radius and the surface tension of surface of tension $R_{\mathrm{S}}$ and $\gamma_{\mathrm{S}}$ is of great importance for the study of interfacial phenomena. To do this, the strict expressions of $R_{\mathrm{S}}$ and $\gamma_{\mathrm{s}}$ in terms of the pressure distribution are needed. ${ }^{[3,4]}$ In a recent paper, Kim et al. ${ }^{[5]}$ conducted MD simulations and presented a detailed characterization of the thermophysical properties of nanoscale liquid threads. As the basis of their MD simulations, they derived a couple of expressions of $R_{\mathrm{S}}$ and $\gamma_{\mathrm{S}}$ in terms of the pressure distribution for nanoscale liquid threads. However, this derivation is wrong both in logical structure and physical thought, although the results are correct. In this paper, a correct new derivation for these expressions will be given in Section 2 and the mistakes appearing in the previous derivation will be pointed out in the Appendix.

\section{Derivation of the expressions of $\boldsymbol{R}_{\mathrm{s}}$ and $\boldsymbol{\gamma}_{\mathrm{s}}$ for nanoscale liquid threads}

Consider the liquid thread of a liqud phase $\alpha$ surrounded by a vapor phase $\beta$. There must be a thin transition layer between phase $\alpha$ and phase $\beta$ where the density of phase $\alpha$ changes to the density of phase $\beta$ continuously. According to Gibbs, the physical system we are studying can be described
DOI: $10.1088 / 1674-1056 / 22 / 12 / 126801$

by two bulk phases of constant densities of liqud and vapor, separated by a dividing surface of radius $R$ located in the thin transition layer of the physical system, so $R$ is not unique. For this model system, we have a general Laplace equation ${ }^{[3]}$

$$
p^{\alpha}-p^{\beta}=\frac{\gamma}{R}+\left[\frac{\mathrm{d} \gamma}{\mathrm{d} R}\right],
$$

where $\gamma$ is the surface tension of the dividing surface and $[\mathrm{d} \gamma / \mathrm{d} R]$ is the derivative for the same physical state of the same system.

Neglecting the small difference between the two transverse components of the pressure tensor, the pressure tensor in cylindrical components is given by

$$
\overline{\bar{P}}=p_{\mathrm{N}}(r) \boldsymbol{e}_{\mathrm{r}} \boldsymbol{e}_{\mathrm{r}}+p_{\mathrm{T}}(r)\left[\boldsymbol{e}_{\theta} \boldsymbol{e}_{\theta}+\boldsymbol{e}_{z} \boldsymbol{e}_{z}\right]
$$

where $e_{\mathrm{r}}, e_{\theta}$ and $e_{z}$ are orthogonal unit vectors, and $p_{\mathrm{N}}(r)$ and $p_{\mathrm{T}}(r)$ are the normal and transverse components of the pressure tensor respectively. ${ }^{[3]}$ The mechanical equilibrium condition of pressure tensor $\nabla \cdot \overline{\bar{P}}=0$ gives $^{[5]}$

$$
p_{\mathrm{T}}(r)=r \frac{\mathrm{d} p_{\mathrm{N}}(r)}{\mathrm{d} r}+p_{\mathrm{N}}(r) .
$$

Equation (3) gives

$$
\frac{\mathrm{d}}{\mathrm{d} r}\left(r^{2} p_{\mathrm{N}}(r)\right)=r\left(p_{\mathrm{N}}(r)+p_{\mathrm{T}}(r)\right) .
$$

Integrating Eq. (4), we obtain

$$
\left(R^{\beta}\right)^{2} p^{\beta}-\left(R^{\alpha}\right)^{2} p^{\alpha}=\int_{R^{\alpha}}^{R^{\beta}} r\left[p_{\mathrm{N}}(r)+p_{\mathrm{T}}(r)\right] \mathrm{d} r
$$

${ }^{*}$ Project supported by the National Natural Science Foundation of China (Grant No. 11072242) and the Research and Development Program of Science and Technology of Higher Education of Shanxi Province, China (Grant No. 20121029). 
where $R^{\alpha}$ and $R^{\beta}$ are the radii of two cylindrical surfaces inside the liquid and vapor, respectively, and $p^{\alpha}$ and $p^{\beta}$ are pressures inside the liquid and vapor, respectively.

Besides, for the $R$ satisfying $R^{\beta}>R>R^{\alpha}$, we have

$\int_{R^{\alpha}}^{R^{\beta}} 2 r p^{\alpha, \beta}(r, R) \mathrm{d} r=\left(R^{\beta}\right)^{2} p^{\beta}-\left(R^{\alpha}\right)^{2} p^{\alpha}+R^{2}\left(p^{\alpha}-p^{\beta}\right)$,

where $p^{\alpha, \beta}(r, R)$ is a defined function $p^{\alpha, \beta}(r, R)=p^{\alpha}(r<R)$, $p^{\alpha, \beta}(r, R)=p^{\beta}(r>R)$

Combining Eqs. (5) and (6), we obtain

$$
R^{2}\left(p^{\alpha}-p^{\beta}\right)=\int_{R^{\alpha}}^{R^{\beta}} r \mathrm{~d} r\left[2 p^{\alpha, \beta}(r, R)-p_{\mathrm{N}}(r)-p_{\mathrm{T}}(r)\right] .
$$

Integrating Eq. (3) yields

$$
\int_{R^{\alpha}}^{R^{\beta}} p_{\mathrm{T}}(r) \mathrm{d} r=R^{\beta} p^{\beta}-R^{\alpha} p^{\alpha} .
$$

Substituting Eqs. (1) and (8) into

$$
\int_{R^{\alpha}}^{R^{\beta}} p^{\alpha, \beta}(r, R) \mathrm{d} r=R^{\beta} p^{\beta}-R^{\alpha} p^{\alpha}+R\left(p^{\alpha}-p^{\beta}\right),
$$

we obtain

$$
p^{\alpha}-p^{\beta}=\frac{\gamma}{R}+\left[\frac{\mathrm{d} \gamma}{\mathrm{d} R}\right]=\frac{1}{R} \int_{R^{\alpha}}^{R^{\beta}}\left[p^{\alpha, \beta}(r, R)-p_{\mathrm{T}}(r)\right] \mathrm{d} r .
$$

Equation (9) is a first-order ordinary differential equation for $\gamma(R)$. It can be written as

$$
\begin{aligned}
p^{\alpha}-p^{\beta}= & \frac{\gamma}{R}+\left[\frac{\mathrm{d} \gamma}{\mathrm{d} R}\right]=\frac{1}{R^{2}} \int_{R^{\alpha}}^{R^{\beta}} r\left[p^{\alpha, \beta}(r, R)-p_{\mathrm{T}}(r)\right] \mathrm{d} r \\
& -\frac{1}{R^{2}} \int_{R^{\alpha}}^{R^{\beta}}(r-R)\left[p^{\alpha, \beta}(r, R)-p_{\mathrm{T}}(r)\right] \mathrm{d} r .
\end{aligned}
$$

We can prove that

$$
\gamma=\frac{1}{R} \int_{R^{\alpha}}^{R^{\beta}} r\left[p^{\alpha, \beta}(r, R)-p_{\mathrm{T}}(r)\right] \mathrm{d} r
$$

is a special solution of Eq. (10) as follows: from Eq. (11), we obtain

$$
\begin{aligned}
{\left[\frac{\mathrm{d} \gamma}{\mathrm{d} R}\right]=} & -\frac{1}{R^{2}} \int_{R^{\alpha}}^{R^{\beta}} r\left[p^{\alpha, \beta}(r, R)-p_{\mathrm{T}}(r)\right] \mathrm{d} r \\
& +\frac{1}{R} \int_{R^{\alpha}}^{R^{\beta}} r\left[\frac{\mathrm{d}}{\mathrm{d} R} p^{\alpha, \beta}(r, R)\right] \mathrm{d} r \\
= & -\frac{1}{R^{2}} \int_{R^{\alpha}}^{R^{\beta}} r\left[p^{\alpha, \beta}(r, R)-p_{\mathrm{T}}(r)\right] \mathrm{d} r+\left(p^{\alpha}-p^{\beta}\right),
\end{aligned}
$$

which, combining with Eq. (9), gives

$$
\left[\frac{\mathrm{d} \gamma}{\mathrm{d} R}\right]=-\frac{1}{R^{2}} \int_{R^{\alpha}}^{R^{\beta}}(r-R)\left[p^{\alpha, \beta}(r, R)-p_{\mathrm{T}}(r)\right] \mathrm{d} r .
$$

Then we obtain Eq. (10).
So the general solution of Eq. (10) is

$$
\gamma=\frac{1}{R} \int_{R^{\alpha}}^{R^{\beta}} r\left[p^{\alpha, \beta}(r, R)-p_{\mathrm{T}}(r)\right] \mathrm{d} r+\frac{C}{R} .
$$

To determine the constant $C$, we consider the equivalence between the model system and the physical system. For the model system, the axial stress distribution is $p^{\alpha, \beta}(r, R)-$ $\gamma(R) \delta(R-r)$ and the total axial stress of the model system must be equivalent to that of the real physical system, i.e.,

$$
2 \pi \int_{R^{\alpha}}^{R^{\beta}} r\left[p^{\alpha, \beta}(r, R)-\gamma(R) \delta(R-r)\right] \mathrm{d} r=2 \pi \int_{R^{\alpha}}^{R^{\beta}} r p_{\mathrm{T}}(r) \mathrm{d} r .
$$

Substituting Eq. (12) into this equation gives $C=0$. Thus the special solution equation (11) is the physical one.

Combining Eq. (7) and Eq. (11) gives

$$
p^{\alpha}-p^{\beta}=\frac{\gamma}{R}+\frac{1}{R^{2}} \int_{R^{\alpha}}^{R^{\beta}} r\left[p^{\alpha, \beta}(r, R)-p_{\mathrm{N}}(r)\right] \mathrm{d} r .
$$

Comparing Eq. (13) with Eq. (1) we obtain

$$
\left[\frac{\mathrm{d} \gamma}{\mathrm{d} R}\right]=\frac{1}{R^{2}} \int_{R^{\alpha}}^{R^{\beta}} r\left[p^{\alpha, \beta}(r, R)-p_{\mathrm{N}}(r)\right] \mathrm{d} r .
$$

The dividing surface with $R=R_{\mathrm{S}}$ satisfying

$$
\left[\frac{\mathrm{d} \gamma}{\mathrm{d} R}\right]_{R=R_{\mathrm{s}}}=0
$$

is called surface of tension. ${ }^{[3]}$ The combination of Eqs. (14) and (15) gives

$$
\int_{R^{\alpha}}^{R^{\beta}} r p^{\alpha, \beta}\left(r, R_{\mathrm{s}}\right) \mathrm{d} r=\int_{R^{\alpha}}^{R^{\beta}} r p_{\mathrm{N}}(r) \mathrm{d} r .
$$

From Eqs. (16) and (11), we obtain

$$
\gamma_{\mathrm{S}}=\frac{1}{R_{\mathrm{S}}} \int_{R^{\alpha}}^{R^{\beta}} r\left[p_{\mathrm{N}}(r)-p_{\mathrm{T}}(r)\right] \mathrm{d} r .
$$

From Eqs. (17) and (3), we obtain

$$
\gamma_{\mathrm{S}}=-\frac{1}{R_{\mathrm{S}}} \int_{R^{\alpha}}^{R^{\beta}} r^{2} \frac{\mathrm{d} p_{\mathrm{N}}(r)}{\mathrm{d} r} \mathrm{~d} r=-\frac{1}{R_{\mathrm{S}}} \int_{0}^{\infty} r^{2} \frac{\mathrm{d} p_{\mathrm{N}}(r)}{\mathrm{d} r} \mathrm{~d} r,
$$

from which, by using general Laplace Eq. (1) and Eq. (15), we obtain

$$
R_{\mathrm{S}}^{2}=-\frac{1}{p^{\alpha}-p^{\beta}} \int_{0}^{\infty} r^{2} \frac{\mathrm{d} p_{\mathrm{N}}(r)}{\mathrm{d} r} \mathrm{~d} r
$$

and

$$
\gamma_{\mathrm{s}}^{2}=-\left(p^{\alpha}-p^{\beta}\right) \int_{0}^{\infty} r^{2} \frac{\mathrm{d} p_{\mathrm{N}}(r)}{\mathrm{d} r} \mathrm{~d} r .
$$

\section{Conclusion}

A couple of expressions of the radius and the surface tension of surface of tension $R_{\mathrm{S}}$ and $\gamma_{\mathrm{s}}$ in terms of the pressure distribution for nanoscale liquid threads are derived. The logic and physical mistakes appearing in the derivation of the same expressions in Ref. [5] are pointed out. 


\section{Acknolegment}

The authors would like to thank professor Zhao Kai-Hua for his valuable suggestion.

\section{Appendix A}

We pointed out the mistakes in the derivation of expressions of $R_{\mathrm{S}}$ and $\gamma_{\mathrm{s}}$ for nanoscale liquid threads in Ref. [5] in the following.

In Appendix A of Ref. [5], a liquid thread was considered. In cylindrical coordinates, the authors obtained the expression of the radius $R_{\mathrm{s}}$, i.e., Eq. (A11) and that of the surface tension $\gamma_{s}$, i.e., Eq. (A12), which are the same as our Eq. (19) and Eq. (20), respectively. In Ref. [5], these two expressions were derived "by combining Eqs. (A1), (A3), (A7), and (A10) for $R=R_{\mathrm{s}}$ ". Among these four Eqs., Eq. (A7) contains two equations, i.e., Eqs. (A7a) and (A7b)

$$
\begin{aligned}
& p_{\mathrm{N}}(r)=p^{\alpha, \beta}\left(r, R_{\mathrm{S}}\right), \\
& p_{\mathrm{N}}(r)-p_{\mathrm{T}}(r)=\gamma_{\mathrm{s}} \delta\left(r-R_{\mathrm{S}}\right),
\end{aligned}
$$

where $\delta\left(r-R_{\mathrm{S}}\right)$ is a Dirac's delta function.

In fact, it is wrong to make use of Eqs. (A7a) and (A7b) in the derivation of Eqs. (A11) and (A12). This is because
Eqs. (A7a) and (A7b) are only a special singular solution of Eq. (A6) (i.e., our first order ordinary differential equation Eq. (9)) in Ref. [5] but not its complete solution, so that it is wrong to use this special singular solution to replace Eq. (A6) in the derivation of the expressions (A11) and (A12) containing continuous function $p_{\mathrm{N}}(r)$. Based on such a derivation in Appendix A of Ref. [5], the expressions (A11) and (A12) (i.e., our Eqs. (19) and (20)) can only be looked at as two properties of the special singular solutions (A7a) and (A7b) and have nothing to do with continuous solution $p_{\mathrm{N}}(r)$. This negates the validity of the derivation in Appendix A of Ref. [5].

\section{References}

[1] Weng J G, Park S H, Lukes J R and Tien C L 2000 J. Chem. Phys. 113 5917

[2] Lee J I, Park S H, Kwon O M, Choi Y K and Lee J S 2002 Ksme Int. J. 161477

[3] Rowlinson J S and Widom B 1982 Molecular Theory of Capillarity (New York: Oxford University Press)

[4] Thompson S M, Gubbins K E, Walton J P R B, Chantry R A R and Rowlinson J S 1984 J. Chem. Phys. 81530

[5] Kim B G, Lee J S, Han M H and Park S 2006 Nanosc. Microsc. Therm. 10283 